\title{
FunctSNP: an R package to link SNPs to functional knowledge and dbAutoMaker: a suite of Perl scripts to build SNP databases
}

\author{
Stephen J Goodswen 1,2, Cedric Gondro², Nathan S Watson-Haigh'1 and Haja N Kadarmideen*1
}

\begin{abstract}
Background: Whole genome association studies using highly dense single nucleotide polymorphisms (SNPs) are a set of methods to identify DNA markers associated with variation in a particular complex trait of interest. One of the main outcomes from these studies is a subset of statistically significant SNPs. Finding the potential biological functions of such SNPs can be an important step towards further use in human and agricultural populations (e.g., for identifying genes related to susceptibility to complex diseases or genes playing key roles in development or performance). The current challenge is that the information holding the clues to SNP functions is distributed across many different databases. Efficient bioinformatics tools are therefore needed to seamlessly integrate up-to-date functional information on SNPs. Many web services have arisen to meet the challenge but most work only within the framework of human medical research. Although we acknowledge the importance of human research, we identify there is a need for SNP annotation tools for other organisms.

Description: We introduce an R package called FunctSNP, which is the user interface to custom built species-specific databases. The local relational databases contain SNP data together with functional annotations extracted from online resources. FunctSNP provides a unified bioinformatics resource to link SNPs with functional knowledge (e.g., genes, pathways, ontologies). We also introduce dbAutoMaker, a suite of Perl scripts, which can be scheduled to run periodically to automatically create/update the customised SNP databases. We illustrate the use of FunctSNP with a livestock example, but the approach and software tools presented here can be applied also to human and other organisms.
\end{abstract}

Conclusions: Finding the potential functional significance of SNPs is important when further using the outcomes from whole genome association studies. FunctSNP is unique in that it is the only R package that links SNPs to functional annotation. FunctSNP interfaces to local SNP customised databases which can be built for any species contained in the National Center for Biotechnology Information dbSNP database.

\section{Background}

Whole genome association studies (WGAS) are a set of methods to study common genetic variation across the entire genome of organisms with the purpose of identifying associations between genetic polymorphisms with observable phenotypic variation in traits. Single nucleotide polymorphisms (SNPs) are the most common genetic variant. There are essentially two advances that have allowed WGAS. Firstly, the huge increase in the

* Correspondence: Haja.Kadarmideen@csiro.au

${ }^{1}$ CSIRO Livestock Industries, Davies Laboratory, University Drive, Townsville, QLD 4810, Australia

Full list of author information is available at the end of the article availability of SNPs. For example, the dbSNP database housed by the National Center for Biotechnology Information (NCBI) contains millions of SNPs for many model species. Secondly, the falling cost and rapid improvements in SNP genotyping technology. One of the main outcomes from WGAS is a subset of SNPs at a specified level of statistical significance ( $\mathrm{p}$ - values) and false discovery rates (FDR). These SNPs are currently used in two conventional, albeit alternative, approaches depending on the requirements of the researcher: 1) a molecular biologist usually searches for candidate genes in the region of the significant SNP and then does functional experiments, which could include targeted sequencing; 2) a 
geneticist uses significant SNPs as DNA markers for predicting disease risks, phenotypic performance or breeding value in individuals and may use this information in associated trait selection (marker-assisted selection/ gene-assisted selection).

One of the major challenges for a researcher using WGAS is minimising false positive rates while maintaining the power to identify true positive associations [1]. WGAS relies on linkage disequilibrium (LD) between DNA markers and causal variants. SNPs located near each other on a chromosome are said to be in LD if they tend to be inherited together more often than can be expected by chance [1]. Unlike the SNP chip design for humans that had input from the HapMap project to assist in its SNP selection, the tag SNP selection for most other species is not strictly based on LD knowledge. For many species the LD patterns are still to be conclusively determined and therefore the tag SNPs for the genotyping SNP chip are selected to be evenly distributed [2].

In WGAS, association does not imply causation. The significant SNPs derived from WGAS can be generally classified into 4 types: 1 ) a causal SNP that contributes to variation in the complex trait; 2) a SNP with no known biological effect but in LD to an untagged SNP (not genotyped) that contributes to variation in the complex trait; 3) a SNP with an association only - no known biological effect or linkage to a causal SNP; 4) a SNP not associated with the complex trait (a false positive). Since the type of significant SNP following WGAS is not distinguishable to the researcher, there is a potential to perform functional experiments based on a false-positive SNP and/or use a SNP as a marker that is neither the causal variant nor in LD with the causal variant.

Currently, the only approach to determine if a WGAS derived significant SNP is a true positive and in LD with the causal variant is to replicate the association findings in an independent population of adequate size [1]. Hence, a need exists for SNP annotation tools that can assist a researcher in distinguishing the type of significant SNP. The tool would need to first collect and integrate all existing biological functional information from online resources. Then provide an interface to access, analyse, and present the information so that the researcher may make informed judgments as to whether a SNP could be a causal variant (type 1) or is in LD with a causal variant (type 2).

Table 1 shows a list of the main current bioinformatics tools for SNP annotation. The tools are web-based and predominantly work within the framework of human medical research. In May 2009, dbSNP held genetic polymorphisms for 45 organisms and 15 of the organisms have sequenced genomes. We have developed a unified bioinformatics tool, called FunctSNP, to address the need to provide SNP annotation for other species as well as humans. FunctSNP is an R package which provides a set of functions to query species-specific local relational databases within the widely used statistical programming language $\mathrm{R}$ http://www.r-project.org/.

For many of the bioinformatics tools, third party applications are required for upstream and downstream research analyses, which in most cases require data format changes. The $\mathrm{R}$ environment has a global data currency of vectors and data frames so there are few data conversion concerns between $\mathrm{R}$ packages. For instance, there are two R packages called GenABEL [3] and SNPassoc [4], which perform genome wide association studies. FunctSNP ideally complements these R packages by linking their output to SNP annotation. In addition, there are R packages called GSA [5], GoSim [6], GeneNet [7], and snp.plotter [8] for further downstream analysis to provide advanced algorithms, and coloured graphs.

A primary goal of FunctSNP is to help screen and select the WGAS significant SNPs which are more likely to be reliable as DNA markers. WGAS makes the direct association between SNP variation and phenotype variation. Whereas in reality SNP variation affects phenotype variation through the effects it has on multiple intervening biological pathways and networks [9]. FunctSNP aims to link WGAS derived SNPs to these biological pathways and gene networks. The FunctSNP functions within $R$ allow screening of SNPs by: 1) determining their physical location with respect to genes. As a general rule, the closer a significant SNP is to the potential causal variant the more likely they will be in LD; 2) finding evidence for a functional role of the significant SNP. It is well known that over $90 \%$ of significant SNPs are not located on genes. Therefore, if the significant SNP is not located on a gene, FunctSNP functions can search in close proximity to the significant SNP for a known SNP (not genotyped) that is on a gene, within a biological pathway, and with a biological function known to be associated with the trait of interest [10]. Figure 1 shows an example, through a schematic diagram, of the process of finding the potential function of significant SNPs.

\section{Construction and content}

The biological information required for SNP annotation is publically available in online databases. Although most of these online resources provide cross-links to other databases, it is not straightforward to extract the relevant research data in one query. Furthermore, even though the databases contain well-annotated data, finding and managing the data of interest among the large assortment of data can be a challenge. Our solution to superfluous and disseminated data was to link SNPs with functional knowledge within species-specific local relational databases. 
Table 1: Existing SNP annotation tools

\begin{tabular}{|c|c|c|c|c|}
\hline Name & Description & Type+ & Species & External Resources Used \\
\hline SNPit [15] & $\begin{array}{l}\text { Analyses the potential functional } \\
\text { significance of SNPs derived from } \\
\text { genome wide association studies. }\end{array}$ & W & Human & $\begin{array}{l}\text { dbSNP, EntrezGene, UCSC Browser, } \\
\text { HGMD, ECR Browser, Haplotter, SIFT }\end{array}$ \\
\hline SNP Function Portal [16] & $\begin{array}{l}\text { Explores the functional } \\
\text { implication of SNP alleles }\end{array}$ & WD & Human & $\begin{array}{l}\text { dbSNP, UniSTS, NCBI ideogram, } \\
\text { Entrez Gene, NCBI human genome } \\
\text { assembly, HapMap Il project }\end{array}$ \\
\hline Pupasuite [17] & SNP prioritization and characterisation & WD & $\begin{array}{l}\text { Human } \\
\text { Mouse } \\
\text { Rat }\end{array}$ & Ensembl, Gene Ontology, OMIM \\
\hline SNPnexus [18] & $\begin{array}{l}\text { Provides functional annotation for both } \\
\text { novel and public SNPs }\end{array}$ & WD & Human & $\begin{array}{l}\text { NCBI RefSeq, UCSC Known Genes, } \\
\text { VEGA, AceView }\end{array}$ \\
\hline LS-SNP/PDB [19] & $\begin{array}{l}\text { Annotates non-synonymous SNPs } \\
\text { mapped to Protein Data Bank structures }\end{array}$ & WD & Human & $\begin{array}{l}\text { UniProtKB, Genome Browser, dbSNP, } \\
\text { PDB }\end{array}$ \\
\hline F-SNP [20] & $\begin{array}{l}\text { Computationally predicts functional } \\
\text { SNPs for disease association studies }\end{array}$ & WD & Human & $\begin{array}{l}\text { PolyPhen, SIFT, SNPeffect, SNPs3D, } \\
\text { LS-SNP, ESEfinder, RescueESE, } \\
\text { ESRSearch, PESX, Ensembl, TFSearch, } \\
\text { Consite, GoldenPath, Ensembl, } \\
\text { KinasePhos, OGPET, Sulfinator, } \\
\text { GoldenPath }\end{array}$ \\
\hline FANS [21] & Functional analysis of novel SNPs & WD & $\begin{array}{l}\text { Human } \\
\text { Mouse }\end{array}$ & $\begin{array}{l}\text { NCBI, Ensembl, UCSC BLAT, Rescue- } \\
\text { ESE } \\
\text { Fas-ESS, SIFT }\end{array}$ \\
\hline SNPer [22] & $\begin{array}{l}\text { Facilitates the retrieval and use of Human } \\
\text { SNPs for high-throughput research } \\
\text { purposes. }\end{array}$ & WD & Human & $\begin{array}{l}\text { dbSNP, Goldenpath, } \\
\text { LocusLink, GeneOntology, SWISS- } \\
\text { PROT, OMIM, Unigene }\end{array}$ \\
\hline SNAP [23] & An integrated SNP annotation platform & W & Human & $\begin{array}{l}\text { Ensembl, UCSC, Uniprot, UniProt, } \\
\text { Pfam, DAS-CBS, MINT, BIND, KEGG, } \\
\text { TreeFam }\end{array}$ \\
\hline SNPeffect [13] & $\begin{array}{l}\text { Predicts the effect of non-synonymous } \\
\text { SNPs on the molecular phenotype of } \\
\text { proteins. }\end{array}$ & W & Human & Ensembl human databases \\
\hline SNPs3D [24] & $\begin{array}{l}\text { Assigns molecular functional effects of } \\
\text { non-synonymous SNPs based on } \\
\text { structure and sequence analysis. }\end{array}$ & W & Human & $\begin{array}{l}\text { dbSNP, SWIIS-Prot, RefSeq, } \\
\text { Bioscience, Gene Ontology, KEGG, } \\
\text { BIND, OMIM, HGMD, Entrez Gene }\end{array}$ \\
\hline
\end{tabular}

+Type = Application type.

$W=$ Web interface to external resources: $W D=$ Web interface to custom database.

\section{Database creation}

We have made available 5 species-specific databases for cattle, chicken, sheep, pig, and human. Each database contains the most pertinent SNP related data extracted from 7 online resources (Table 2). We use the public domain relational database management system (RDMS) SQLite http://www.sqlite.org/. It stores data in a file that is platform independent, provides batch mode functionality, and is the preferred RDMS for R. The data contained in the online resources is dynamic, so we have developed a suite of Perl scripts, collectively called dbAutoMaker, to provide a framework in which to automate the process of creating a local database with data extracted from any number of resources. Figure 2 shows the 4 automated steps performed by dbAutoMaker: 1 ) download - reads, from a configuration file, a list of Uniform Resource Locators (URLs) associated with the online resource data files and then downloads them; 2) decompress - automatically decompresses files with suffixes tar, tgz, gz, and zip; 3) convert - converts the data into an SQL format that can be imported to a SQLite database. There is currently no standardized interchange format for transporting data from one database to the next. The data formats encountered were Microsoft SQL Server, MySQL, XML, and ASCII text file (flat file). Generic Perl scripts that work in conjunction with configuration files were developed to handle each data format; 4) import - imports the data into the SQLite database 

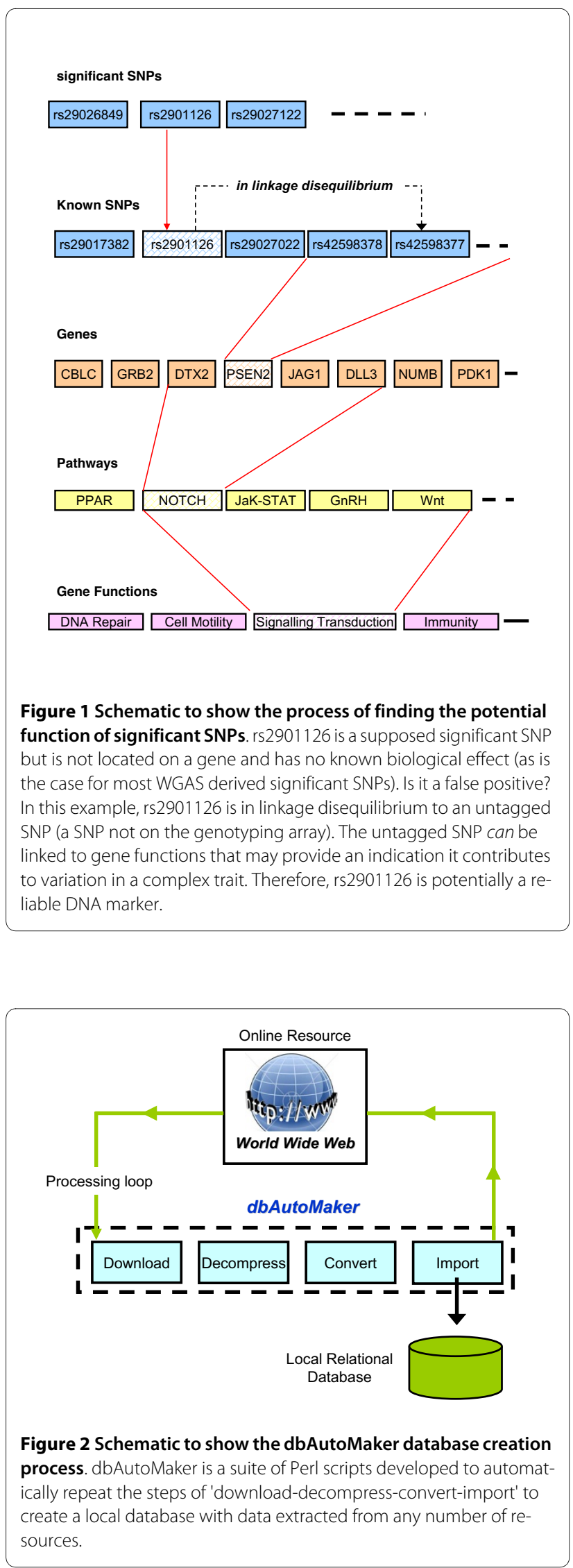

using SQLite batch mode command files created by the Perl scripts in step 3 . These 4 steps are repeated for each online resource. dbAutoMaker can be scheduled to run periodically to ensure the local database is always up-todate. No further intervention is required once the framework and schedule is setup.

The core of dbAutoMaker is a species-specific configuration file in an INI file format. Although we have demonstrated dbAutoMaker using mainly livestock species, it can be configured by the user to create a SNP database for any species contained in NCBI's dbSNP [for more detail refer to dbAutoMaker User Guide on http:// www.csiro.au/science/dbAutoMaker.html].

Figure 3 shows the schema for the SNP relational database. Each table can be linked through the SNP_ID or Gene_ID attributes and consequently all data is integrated.

The SQLite databases can be downloaded from http:// www.csiro.au/science/FunctSNP.html. The user can directly query the database using Structured Query Language (SQL) through the SQLite program. However, we recommend FunctSNP as the database user interface.

The quality of results that can be expected from FunctSNP is reliant on the amount and reliability of information extracted from the online resources. For example, based on the amount of data available, the Homo sapiens database is information rich in comparison to all other species. Bos taurus and Gallus gallus databases are poor in comparison to Homo sapiens but substantially richer compared to Sus scrofa and Ovis aries. The table in Additional file 1 is a summary of the number of entries in the FunctSNP species-specific databases that were extracted from the online resources. Given time it is expected that the amount of available data for all species will dramatically increase.

\section{Utility}

FunctSNP provides its utility through $\mathrm{R}$ functions. So far, $20 \mathrm{R}$ functions (Table 3) have been implemented for analysing the biological information. Although FunctSNP is primarily designed to link WGAS derived significant SNPs to biological information, it is flexible and there is no restriction on how, or what order, the R functions can be used. The FunctSNP functions are described in full in the manual pages supplied with the R package, so are only briefly mentioned here.

The FunctSNP functions provide access to the biological information contained within species-specific databases. Information such as: SNP chromosomal location, exon/intron status, synonymous/non-synonymous effect, SNPs in Quantitative Trait Loci (QTL) regions, biological pathways, GO terms, and protein products for related genes. Multiple databases (one for each species) can be queried in the same R session. 
Table 2: External resources used to create SNP customised database

\begin{tabular}{|c|c|c|c|}
\hline Acronym & Name & Link & Resource \\
\hline dbSNP & $\begin{array}{l}\text { Single Nucleotide Polymorphism } \\
\text { database }\end{array}$ & http://www.ncbi.nlm.nih.gov/ & SNPs \\
\hline GO & Gene Ontology & http://www.geneontology.org/ & Genes and gene product attributes \\
\hline KEGG & $\begin{array}{l}\text { Kyoto Encyclopaedia of Genes and } \\
\text { Genomes }\end{array}$ & http://www.genome.jp/kegg/ & Biological pathways \\
\hline UniProt & Universal Protein Resource & http://www.uniprot.org/ & $\begin{array}{l}\text { Protein sequences and functional } \\
\text { information }\end{array}$ \\
\hline $\mathrm{QTLdb}^{++}$ & $\begin{array}{l}\text { Animal Quantitative Trait Locus } \\
\text { database }\end{array}$ & $\begin{array}{l}\text { http://www.animalgenome.org/ } \\
\text { QTLdb/ }\end{array}$ & Quantitative Trait Loci data (QTL) \\
\hline $\mathrm{OMIA}^{++}$ & $\begin{array}{l}\text { Online Mendelian Inheritance in } \\
\text { Animals }\end{array}$ & http://omia.angis.org.au/ & Genes, inherited disorders and traits \\
\hline HomoloGene & & $\begin{array}{l}\text { http://www.ncbi.nlm.nih.gov/ } \\
\text { homologene }\end{array}$ & Homolog detection \\
\hline
\end{tabular}

${ }^{++}$Not applicable to Homo sapiens

Central to the SNP databases are the SNP ID (NCBI's dbSNP rs\# cluster ID) and gene ID (NCBI's gene ID). Consequently, the majority of FunctSNP functions accept, as input, a vector of either SNP IDs or gene IDs by specifying what type of IDs are being passed to the function using id.type. For example:

snps <- getSNPs (ids,id.type = "snp")

The getSNPs () function returns SNP information (e.g., chromosome number, chromosomal location, protein coding status etc.) for each SNP ID in the ids vector.

snps <- getSNPs (ids,id.type = "gene")

This time, the getSNPs () function returns SNP information for all SNPs residing within each of the genes specified by their gene IDs in the ids vector.

As a general guideline to the user in prioritising the SNPs most likely to have significant functional roles, all SNPs in the database have been scored. The total score is comprised of a simple sum of two components. The first component is a score according to the SNP location and the type of amino acid alteration it may cause. We use NCBI's dbSNP function classification for SNPs on genes. The table in Additional file 2 shows the function classification in the first column and the FunctSNP score value in the second column. The classification is ranked and scored according to the likelihood of the function type altering the amino acid. For example, a SNP is given a maximum score if the polymorphism alters an amino acid. We acknowledge that scoring is subjective and therefore it is our intention in a future version of FunctSNP to allow a user-defined score value for each function classification. The second component used in the calculation of the total score is a cumulative score according to the amount of supporting biological information found in the database. If the SNP can be linked to proteins, GO terms, KEGG pathways, QTL regions, and homologous genes, the score is incremented for each linkage. For example, a SNP assigned a score of 26 (the maximum possible score) is derived as follows: 20 (SNP alters codon to make an altered amino acid) +1 (SNP is located in a QTL region) + 1 (Protein information found) +1 (one or more GO terms found) + 1 (one or more KEGG pathways found) + 1 (one or more OMIA information found) +1 (homologous genes found).

There are $\mathrm{R}$ functions to: find the nearest genes to each SNP ID entered, using a chromosomal base pairs search distance - getGenesByDist(); find the nearest high scoring SNP to each SNP ID, or chromosomal location entered getHighScoreSNP(); find homologous genes across all species or a specified species for each SNP ID, or gene ID entered - getHomolo(); find the quantitative trait loci (QTL) region associated to a trait (as annotated within QTLdb database) for each SNP ID or gene ID entered getTraits(); and extract SNP ID for each chromosomal location entered - getSNPID(). There are also R functions to download the latest customised SNP database for a particular species (currently, databases for cattle, chicken, sheep, pig, and human are available) - downloadDB(); and a function to invoke dbAutoMaker for the user to make their own databases - makeDB().

\section{Program testing}

To test FunctSNP, we used $10 \mathrm{k}$ SNP genotype data from a yet to be published livestock WGAS. From this $10 \mathrm{k}$ SNP data, 165 SNPs had been identified as being significantly associated with a particular trait of economic interest. These SNPs were sufficiently quality controlled and accepted as reliable prior to using FunctSNP. 


Hom ologene
Gene_Set INT
Taxon_ID INT
Gene_ID INT
Gene_Symbol VARCHAR(20)
Protein_GI INT
Protein_ID VARCHAR(20)
Taxon_Name TEXT

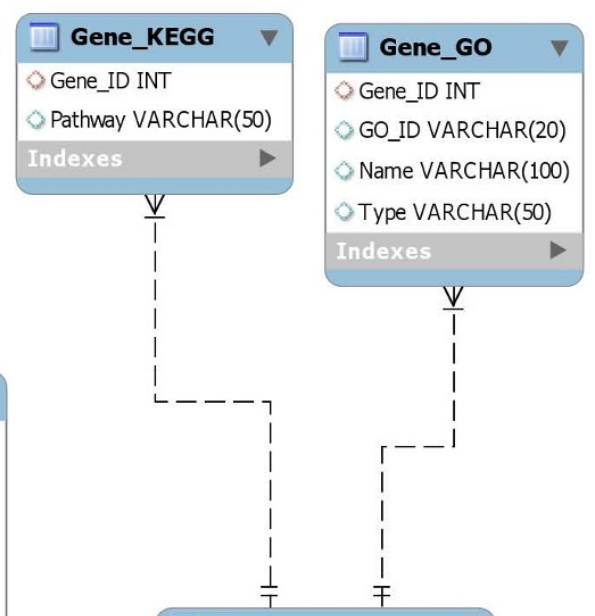

Characterised VARCHAR(10)

Marker VARCHAR(20)

Symbol VARCHAR(20)

Inherit_Name VARCHAR(30)

Phene_Name VARCHAR(100)

$\triangle$ Clin_Feat TEXT

Map_Info TEXT

History TEXT

$\triangle$ Pathology TEXT

Prevalence TEXT

Mol_Gen TEXT

Control TEXT

Gen_Test TEXT

Summary TEXT

Indexes
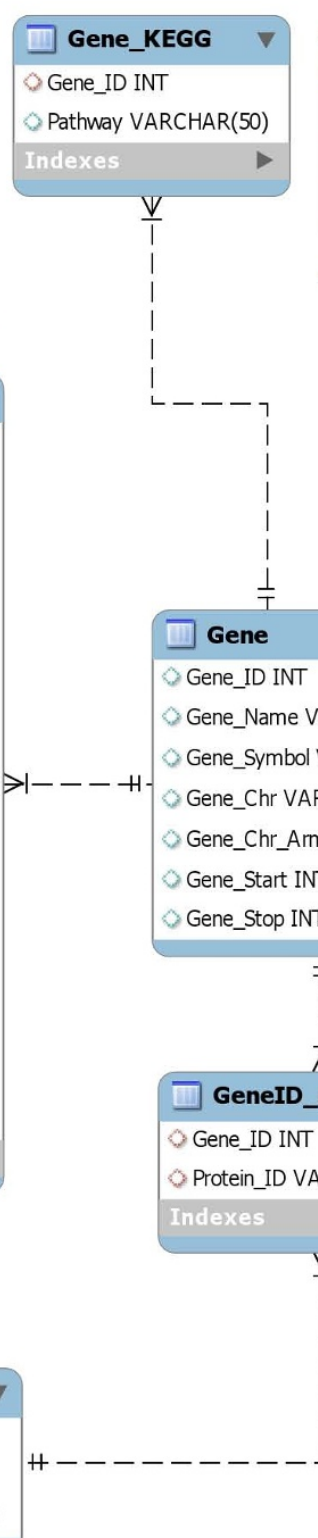
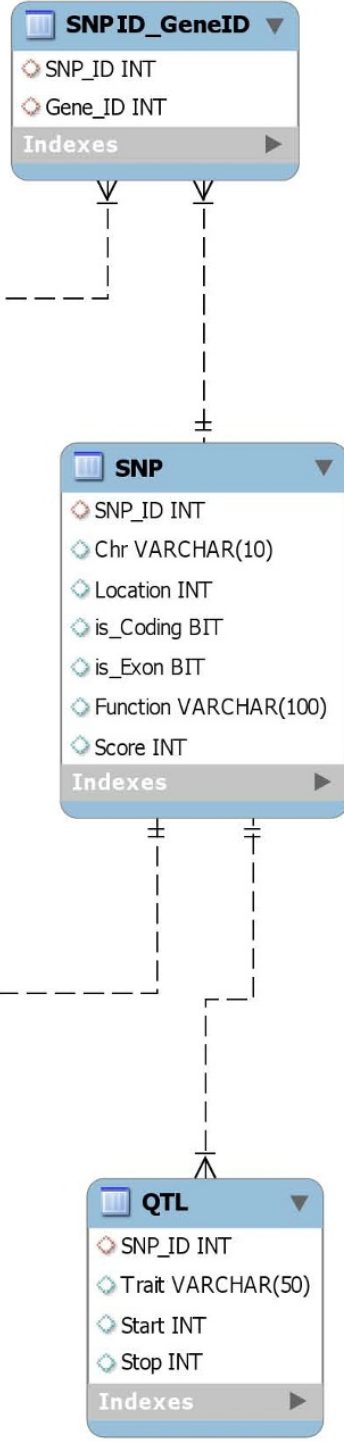

Figure 3 Database Schema for custom built SNP database 
Table 3: FunctSNP R Functions

\begin{tabular}{|c|c|}
\hline Name & Description \\
\hline addSpecies & $\begin{array}{l}\text { Adds a new species to the list of species } \\
\text { recognized by FunctSNP }\end{array}$ \\
\hline downloadDB & $\begin{array}{l}\text { Download pre-assembled species- } \\
\text { specific databases }\end{array}$ \\
\hline getGenelD & $\begin{array}{l}\text { Extract gene ID information using SNP } \\
\text { IDs or SNP locations }\end{array}$ \\
\hline getGenes & $\begin{array}{l}\text { Extract gene information using SNP IDs } \\
\text { or Gene IDs }\end{array}$ \\
\hline getGenesByDist & $\begin{array}{l}\text { Extract gene ID within a specified } \\
\text { distance from a SNP }\end{array}$ \\
\hline getGO & $\begin{array}{l}\text { Extract gene ontology using SNP IDs or } \\
\text { Gene IDs }\end{array}$ \\
\hline getHighScoreSNP & $\begin{array}{l}\text { Extract highest scoring SNP using SNP } \\
\text { IDs or SNP locations }\end{array}$ \\
\hline getHomolo & $\begin{array}{l}\text { Extract homologous genes using SNP } \\
\text { IDs or Gene IDs }\end{array}$ \\
\hline getKEGG & $\begin{array}{l}\text { Extract pathway names using SNPIDs or } \\
\text { Gene IDs }\end{array}$ \\
\hline getNearGenes & $\begin{array}{l}\text { Find nearest genes to either SNP IDs or } \\
\text { SNP locations }\end{array}$ \\
\hline getOMIA & Extract OMIA using SNP IDs or Gene IDs \\
\hline getProteins & $\begin{array}{l}\text { Extract protein information using SNP } \\
\text { IDs or Gene IDs }\end{array}$ \\
\hline getSNPID & $\begin{array}{l}\text { Extract SNP ID using Gene IDs or SNP } \\
\text { locations }\end{array}$ \\
\hline getSNPs & $\begin{array}{l}\text { Extract SNP information using SNP IDs } \\
\text { or Gene IDs }\end{array}$ \\
\hline getTraits & $\begin{array}{l}\text { Extract traits associated with QTL } \\
\text { regions using SNP IDs or Gene IDs }\end{array}$ \\
\hline installedDBs & Displays the local available databases \\
\hline makeDB & $\begin{array}{l}\text { Build a species-specific database from } \\
\text { external sources }\end{array}$ \\
\hline setSpecies & Sets the default species \\
\hline supportedSpecies & Displays the supported species \\
\hline userAddedSpecies & Displays the species added by user \\
\hline
\end{tabular}

The aim of the test was to rank the 165 SNPs according to their likelihood of being reliable DNA markers. Our premise for reliable DNA markers is that WGAS derived SNPs should be either the causal variant or close to a SNP that is the causal variant. In FunctSNP, whether a SNP is a potential causal variant is determined by its assigned functional role and its links to biological information (e.g., pathways and gene ontology). The SNPs in the database have been scored in accordance with this premise. Although, high scoring SNPs in FunctSNP are the most likely to have significant functional roles, the expertise of the researcher is required to make that important mental link between the biological information provided and its relevance to the trait of interest. We also use the general rule that the closer a WGAS derived SNP is to a high scoring SNP the more likely the SNPs will be in LD. However, the researcher needs to be aware that this rule does not always hold true as there are blocks of SNPs along the chromosome with higher and lower degrees of LD. For example, for the most part the closer 2 SNPs are together the stronger the LD is going to be, nevertheless, it is possible that the 2 SNPs are located on different blocks of LD.

The test involved 4 different runs based on the following criteria that determined the data input to the FunctSNP functions: 1) using all 165 WGAS derived significant SNPs; 2) using only significant SNPs with a score greater than 7; 3) using SNPs with a score greater than 11 from the database that are within 10,000 base pairs from the 165 significant SNPs; and 4) using SNPs from the database with a score greater than 24 that are located on genes within 10,000 base pairs from the 165 significant SNPs. The threshold score used is at the discretion of the user. SNPs with a score equal or greater than 20 are considered highly likely to have significant functional roles. The base pairs search distance is also subjective. Knowledge of the LD patterns for the research species will need to govern the distance used.

Table 4 is a summary of the amount of information obtained from each test criterion. In run 1, all 165 of the significant SNPs were input into the functions getGenes(), getProteins(), getKEGG(), getGO(), and getHomolo (). Although, the SNPs are located on 52 genes which can be linked to 23 proteins, 43 biological pathways, and 259 GO terms only 1 significant SNP could be considered a candidate causal variant. We did not analyse the output from this run any further and proceeded with test run 2. In order to prune the output information this run used significant SNPs with a score greater than 7 as input to functions. The GO IDs, KEGG pathway names, and UniProt IDs that were output from run 2, 3 and 4 were used as input to their associated websites to find additional clues (a step that we recommend in the FunctSNP demonstration guide located at http:// www.csiro.au/science/FunctSNP.html). For example, GO IDs e.g., GO: 0001875, can be input under "Search the Gene Ontology Database" at http://www.geneontology.org/; KEGG pathway names e.g., path:bta04620, input "bta" under "Select Prefix" and "04620" under "Enter Keywords" at http://www.genome.jp/kegg/pathway.html; and UniProt IDs e.g., Q4GZT3, input under UniProt homepage at http://www.uniprot.org/.

The purpose of run 3 was to find the highest scoring SNPs in the database residing in close proximity to the significant SNPs. We found 15 SNPs located on 15 different genes and 8 of these SNPs were considered to have significant functional roles. Similarly, the purpose of run 
Table 4: Shows a summary of the output count after 4 program test runs

\begin{tabular}{|c|c|c|c|c|c|c|c|c|}
\hline \multirow[b]{2}{*}{ \# } & \multicolumn{3}{|c|}{ Number of SNPs ... } & \multicolumn{5}{|c|}{ Number of entries found in database for SNPs meeting input criteria ${ }^{++}$} \\
\hline & $\begin{array}{l}\text { meeting input } \\
\text { criteria } \$ \$\end{array}$ & $\begin{array}{l}\text { derived from } \\
\text { WGAS meeting } \\
\text { criteria }\end{array}$ & $\begin{array}{l}\text { found in database } \\
\text { with score }=>20\end{array}$ & Genes & Proteins & Pathways & Gene Ontology & $\begin{array}{l}\text { Homologous } \\
\text { Genes(with Homo } \\
\text { sapiens) }\end{array}$ \\
\hline 1 & 165 & 165 & 1 & 52 & 23 & 43 & 259 & 47 \\
\hline 2 & 7 & 7 & 1 & 7 & 4 & 19 & 29 & 5 \\
\hline 3 & 15 & 1 & 8 & 15 & 9 & 29 & 114 & 13 \\
\hline 4 & 11 & 0 & 11 & 6 & 10 & 21 & 126 & 6 \\
\hline & $\begin{array}{l}=\text { test run number } \$ \$ \\
\text { all } 165 \text { significant SI } \\
\text { significant SNPs wit } \\
\text { SNPs with score gre } \\
\text { SNP with score grea } \\
\text { Example: For run } 3 \text {, } \\
\text { t the criteria of scor } \\
15 \text { genes with } 9 \text { pro } \\
\text { nes. }\end{array}$ & $\begin{array}{l}\text { riteria determines } \\
\text { Ps } \\
\text { score greater than } \\
\text { ter than } 11 \text { within } \\
\text { er than } 24 \text { located } \\
\text { e highest scoring } \\
>11 ; 1 \text { out of the } \\
\text { ein products. The } g\end{array}$ & $\begin{array}{l}7 \\
0,000 \text { base pairs from } \\
\text { on genes within } 10,00 \\
\text { NPs within } 10,000 \text { ba } \\
15 \text { SNPs found was a } \\
\text { enes are involved in } 2\end{array}$ & $\begin{array}{l}\text { significa } \\
\text { base pa } \\
\text { e pairs fr } \\
\text { VGAS der } \\
\text { pathway }\end{array}$ & $\begin{array}{l}\text { t SNPs } \\
\text { s from sign } \\
\text { m } 165 \text { sign } \\
\text { led SNP; } 8 \text { S } \\
\text {, have } 114 \text { C }\end{array}$ & $\begin{array}{l}\text { icant SNPs. } \\
\text { icant SNPs we } \\
\text { NPs from the } \\
\text { ene Ontology }\end{array}$ & $\begin{array}{l}\text { re found in databas } \\
5 \text { have a score => } \\
\text { terms, and are hom }\end{array}$ & $\begin{array}{l}\text {; } 15 \text { of the found SNPs } \\
0.15 \text { SNPs are located } \\
\text { logous with } 13 \text { human }\end{array}$ \\
\hline
\end{tabular}

4 was to find genes in close proximity to the significant SNPs and then find the highest scoring SNPs residing on these genes. We found 6 genes in close proximity that contained 11 SNPs with significant functional roles. Based on the results of the 4 tests we were able to rank the 165 SNPs according to their likelihood of being reliable DNA markers and concluded that the top 8 were realistic candidate DNA markers. In particular, 2 WGAS derived SNPs were identified to be in close proximity to 2 genes influencing biological pathways that literature have linked to the WGAS trait of interest. See additional file 3 for FunctSNP commands used in testing program.

\section{Discussion}

As part of the ongoing development, we will monitor web resources for new and improved sources of data that can be incorporated into FunctSNP. The design of dbAutoMaker allows data to be extracted from new online resources with minimal modification to dbAutoMaker's configuration file.

FunctSNP and our approach for identifying SNP markers currently make the assumption that all causal SNPs are located on genes. There are now many suggestions that non coding SNPs (e.g., SNPs in transcription factor binding sites, splice sites, repressor, promoter regions, and eQTLs [9]) could significantly contribute to variation in complex traits. Therefore, as part of the ongoing development, online resources (such as coliSNP [11], TRANSFAC [12], SNPeffect [13], eQTL Explorer [14]) are being investigated to find data for the functional role of non- coding SNPs with the intention of incorporating this data in FunctSNP databases.

\section{Conclusions}

To take full advantage of WGAS, we need to make that essential link between the outcomes from WGAS and the information that exists about the function of genes and pathways. Whilst there are many emerging bioinformatics tools to make this link, we have developed a tool called FunctSNP which we believe is unique and addresses some of the shortcomings of existing tools. Firstly, FunctSNP is the only $\mathrm{R}$ package designed specifically for SNP annotation. There are considerable advantages over a web interface in integrating FunctSNP within the $\mathrm{R}$ statistical computing environment offering high quality graphics and data visualization tools. Operating in the same $R$ environment, FunctSNP has notable complementary upstream and downstream research $\mathrm{R}$ packages. Secondly, the purpose built database for FunctSNP can include information about SNPs in other organisms besides human. We have developed an application called dbAutoMaker for a researcher to automatically build their own SNP database for any species contained in NCBI's dbSNP. We have scheduled dbAutoMaker to build databases for cattle, chicken, pig, sheep, and human on a monthly basis. These databases can easily be downloaded whenever required directly from the web or using a FunctSNP function.

We conclude that FunctSNP is a post-WGAS tool that provides an opportunity to screen and select for SNPs 
that have a higher likelihood to be related to variation in a particular complex trait of interest.

\section{Availability and requirements}

The FunctSNP R package is available from CRAN:

http://cran.r-project.org/package=FunctSNP.

In

FunctSNP there are no new classes, no overloading, and default storage modes for R objects are used.

The software for dbAutoMaker is implemented in Perl and supported on Linux and MS Windows. Databases and source code are freely available from: http:// www.csiro.au/science/FunctSNP.html and http:// www.csiro.au/science/dbAutoMaker.html

\section{Additional material}

Additional file 1 Table - Summary of the number of entries in the customised species-specific SNP databases.

Additional file 2 Table - FunctSNP SNP prioritisation.

Additional file 3 FunctSNP commands used in testing program.

\section{Authors' contributions}

HNK conceived this systems genetics project, and developed and obtained the CSIRO research grant for the project. SJG, CG and NSW developed FunctSNP. SJG developed dbAutoMaker. CG and HNK supervised the project. All 4 authors wrote the paper, read and approved the final manuscript.

\section{Acknowledgements}

Office of the Chief Executive (OCE) of CSIRO for postgraduate scholarship. Special thanks to Professor Julius van der Werf (University of New England) who provided helpful guidance and manuscript editing.

\section{Author Details}

${ }^{1}$ CSIRO Livestock Industries, Davies Laboratory, University Drive, Townsville, QLD 4810, Australia and 2Centre for Genetic Analysis and Applications, University of New England, Armidale, NSW 2351, Australia

Received: 21 March 2010 Accepted: 9 June 2010

Published: 9 June 2010

\section{References}

1. Pearson TA, Manolio TA: How to interpret a genome-wide association study. JAMA 2008, 299(11):1335-1344

2. Wall JD, Pritchard JK: Haplotype blocks and linkage disequilibrium in the human genome. Nature Reviews Genetics 2003, 4(8):587-597.

3. Aulchenko YS, Ripke S, Isaacs A, van Duijn CM: GenABEL: an R library for genome-wide association analysis. Bioinformatics 2007 23(10):1294-1296

4. Gonzalez JR, Armengol L, Sole X, Guino E, Mercader JM, Estivill X, Moreno $\mathrm{V}$ : SNPassoc: an R package to perform whole genome association studies. Bioinformatics 2007, 23(5):654-a-655.

5. Subramanian A, Tamayo P, Mootha VK, Mukherjee S, Ebert BL, Gillette MA, Paulovich A, Pomeroy SL, Golub TR, Lander ES, et al:: Gene set enrichment analysis: A knowledge-based approach for interpreting genome-wide expression profiles. Proc Natl Acad Sci USA 2005, 102(43):15545-15550.

6. Frohlich H, Speer N, Poustka A, Beissarth T: GOSim - an R-package for computation of information theoretic $\mathrm{GO}$ similarities between terms and gene products. BMC Bioinformatics 2007:8.

7. Opgen-Rhein R, Strimmer K: From correlation to causation networks: a simple approximate learning algorithm and its application to highdimensional plant gene expression data. Bmc Systems Biology 2007:1.

8. Luna A, Nicodemus KK: snp.plotter: an R-based SNP/haplotype association and linkage disequilibrium plotting package. Bioinformatics 2007, 23(6):774-776.
9. Kadarmideen HN: Genetical systems biology in livestock: application to gonadotrophin releasing hormone and reproduction. let Systems Biology 2008, 2(6):423-441.

10. Maher B: Personal genomes: The case of the missing heritability. Nature 2008, 456(7218):18-21.

11. Kono H, Yuasa T, Nishiue S, Yura K: coliSNP database server mapping nsSNPs on protein structures. Nucl Acids Res 2008 36(suppl_1):D409-413.

12. Matys V, Fricke E, Geffers R, Gossling E, Haubrock M, Hehl R, Hornischer K Karas D, Kel AE, Kel-Margoulis OV, et al:: TRANSFAC (R): transcriptional regulation, from patterns to profiles. Nucleic Acids Res 2003, 31(1):374-378

13. Reumers J, Maurer-Stroh S, Schymkowitz J, Rousseau F: SNPeffect v2.0: a new step in investigating the molecular phenotypic effects of human non-synonymous SNPs. Bioinformatics 2006, 22(17):2183-2185.

14. Mueller M, Goel A, Thimma M, Dickens NJ, Aitman TJ, Mangion J: eQTL Explorer: integrated mining of combined genetic linkage and expression experiments. Bioinformatics 2006, 22(4):509-511.

15. Shen TH, Carlson CS, Tarczy-Hornoch P: SNPit: A federated data integration system for the purpose of functional SNP annotation. Comput Methods Programs Biomed 2009, 95(2):181-189.

16. Wang PL, Dai MH, Xuan WJ, McEachin RC, Jackson AU, Scott LJ, Athey B, Watson SJ, Meng F: SNP Function Portal: a web database for exploring the function implication of SNP alleles. Bioinformatics 2006, 22(14):E523-E529.

17. Conde L, Vaquerizas JM, Dopazo H, Arbiza L, Reumers J, Rousseau F, Schymkowitz J, Dopazo J: PupaSuite: finding functional single nucleotide polymorphisms for large-scale genotyping purposes. Nucleic Acids Res 2006, 34:W621-W625.

18. Chelala C, Khan A, Lemoine NR: SNPnexus: a web database for functional annotation of newly discovered and public domain single nucleotide polymorphisms. Bioinformatics 2009, 25(5):655-661.

19. Ryan M, Diekhans M, Lien S, Liu Y, Karchin R: LS-SNP/PDB: annotated non-synonymous SNPs mapped to Protein Data Bank structures. Bioinformatics 2009, 25(11):1431-1432.

20. Lee PH, Shatkay H: F-SNP: computationally predicted functional SNPs for disease association studies. Nucleic Acids Res 2008, 36:D820-D824.

21. Liu CK, Chen YH, Tang CY, Chang SC, Lin YJ, Tsai MF, Chen YT, Yao A Functional analysis of novel SNPs and mutations in human and mouse genomes. BMC Bioinformatics 2008:9.

22. Riva A, Kohane IS: A SNP-centric database for the investigation of the human genome. BMC Bioinformatics 2004:5.

23. Li ST, Ma LJ, Li H, Vang S, Hu YF, Bolund L, Wang J: Snap: an integrated SNP annotation platform. Nucleic Acids Res 2007, 35:D707-D710.

24. Yue P, Melamud E, Moult J: SNPs3D: Candidate gene and SNP selection for association studies. BMC Bioinformatics 2006:7.

doi: $10.1186 / 1471-2105-11-311$

Cite this article as: Goodswen et al., FunctSNP: an R package to link SNPS to functional knowledge and dbAutoMaker: a suite of Perl scripts to build SNP databases BMC Bioinformatics 2010, 11:311

\section{Submit your next manuscript to BioMed Centra} and take full advantage of:

- Convenient online submission

- Thorough peer review

- No space constraints or color figure charges

- Immediate publication on acceptance

- Inclusion in PubMed, CAS, Scopus and Google Scholar

- Research which is freely available for redistribution 